\title{
Influence of powder morphology and sintering temperature on the properties of hydroxyapatite
}

\author{
W.J. Kelvin Chew ${ }^{1}$, A. Niakan ${ }^{2}$, N.A. Nawawi ${ }^{2}$, L.T. Bang ${ }^{2}$ and S. Ramesh ${ }^{2 *}$ \\ ${ }^{1}$ School of Engineering, Taylor's University, Taylor's Lakeside Campus, \\ No. 1 Jalan Taylor's, 47500 Subang Jaya, Malaysia. \\ ${ }^{2}$ Center of Advanced Manufacturing and Material Processing, \\ Department of Mechanical Engineering, Faculty of Engineering, \\ University of Malaya, 50603 Kuala Lumpur, Malaysia \\ *Email: ramesh79@um.edu.my \\ Phone: +60379675209; Fax: +60379675330
}

\begin{abstract}
The effect of sintering two hydroxyapatite (HA) powders (designated HAC and HAS) of contrasting morphology, at temperatures ranging from $1000^{\circ} \mathrm{C}$ to $1450^{\circ} \mathrm{C}$, on the phase stability of the material was investigated. Pellets were prepared by cold isostatic pressing of the powders at $200 \mathrm{MPa}$ and subsequently sintering in air at a heating rate of $2^{\circ} \mathrm{C} / \mathrm{min}$. and a holding time of 2 hours. X-ray diffraction analysis revealed that decomposition of the HA was dependent on the morphology of the precursor powders. The decomposition of the HAS powder started to occur at a lower sintering temperature of $1300^{\circ} \mathrm{C}$, whereas the HAC powder only decomposes at $1400^{\circ} \mathrm{C}$. Both powders formed residual phases of tri-calcium phosphates and tetra-calcium phosphate due to decomposition. The possible factors controlling the decomposition mechanism of the HA powders are also discussed.
\end{abstract}

Keywords: Hydroxyapatite (HA); bioceramics; decomposition; morphology.

\section{INTRODUCTION}

Hydroxyapatite, a calcium phosphate based compound with the chemical constituent $\mathrm{Ca}_{10}\left(\mathrm{PO}_{4}\right)_{6}(\mathrm{OH})_{2}$, is the main mineral component of bone and teeth. This material is the most commonly used ceramic for bone replacement due to its superior biocompatibility, which allows for intergrowth of new bones onto the material [1]. However, one of the major factors that limits the full exploitation of HA ceramics as implants is the brittle nature of the material. As a result of its low fracture toughness $\left(0.8\right.$ to $\left.1.2 \mathrm{MPa} . \mathrm{m}^{1 / 2}\right)$ and flexural strength $(<130 \mathrm{MPa})[2,3]$, the application of hydroxyapatite is confined to nonload-bearing members and unstressed regions of the body [3-5]. A great deal of research has been done to control the properties of the precursor powders used to produce HA ceramics, as it is critical in determining the microstructure of the sintered ceramics, which in turn influences the physical and mechanical properties of the material [6-10]. Particle size, shape and agglomeration are some of the main features that control the sinterability of the compacted material. For example, the presence of agglomerates, in particular, of the hard nature, tends to disrupt the densification process and lead to the occurrence of defects in the microstructure, which inevitably limits the strength of the sintered body [11]. Particle size is also an important criterion as it determines the activation energy and hence the grain growth of the ceramic $[8,12]$. These are some of the main critical factors that have been shown to influence the hardness of hydroxyapatite ceramic [12]. The 
objective of this work was to compare two HA powders of different morphologies that were produced via different routes and to relate some of their properties to the sintering characteristics of the material.

\section{EXPERIMENTAL DETAILS}

\section{Synthesis}

In the present work, two powders from different sources were employed. The first powder, designated as HAC, is a commercially available hydroxyapatite powder (Merck 2196, Germany) while the second powder, designated as HAS, was prepared via the aqueous precipitation technique using reagent grade $\mathrm{Ca}(\mathrm{OH})_{2}$ and $\mathrm{H}_{3} \mathrm{PO}_{4}$. The powders were pressed into cylindrical disc samples via uniaxial pressing at $40 \mathrm{MPa}$ using a $20 \mathrm{~mm}$ steel die followed by cold isostatic pressing at $200 \mathrm{MPa}$. The green pellets were sintered in air atmosphere at different temperatures ranging from $1000^{\circ} \mathrm{C}$ to $1450^{\circ} \mathrm{C}$, at a furnace ramp rate of $2^{\circ} \mathrm{C} / \mathrm{min}$ and a holding time of 2 hours. The pellets were subsequently allowed to cool slowly in the furnace until ambient temperature.

\section{Characterization}

The calcium content in the HA powders was determined by the Inductive Coupled Plasma (ICP) method after dissolving in nitric acid, whereas the titration method of standard EDTA solution was used to determine the phosphate content. The density of the sintered compacts was obtained by the water immersion technique and expressed in terms of relative density with the theoretical density of HA taken to be $3.156 \mathrm{gcm}^{-3}$. Phase analysis by $\mathrm{X}$-ray diffraction (XRD) of the samples was performed at room temperature using $\mathrm{Cu}$ $\mathrm{K} \alpha$ as the radiation source. The crystalline phases present in the samples were identified by comparison with standard reference JCPDS (Joint Committee on Powder Diffraction Standards) files. In addition, the microstructural evolution under the various sintering temperatures was examined using a scanning electron microscope (SEM). Prior to SEM analysis, the samples were polished to a $1 \mu \mathrm{m}$ surface finish using diamond paste and subsequently etched with $0.5 \% \mathrm{HF}$ to delineate the grain boundaries. The microhardness of the samples was measured using the Vicker's indentation method. The indentation load was kept constant at $1.96 \mathrm{~N}$ (200 grams) and applied for 10 seconds. The average hardness value was taken from five indents made for each sample and the maximum error obtained was found to be less than $5 \%$.

\section{RESULTS AND DISCUSSION}

\section{Characterization of Powders}

The measured quantities of $\mathrm{Ca}$ and $\mathrm{P}$ in the HAS sample were $37.35 \% \mathrm{w} / \mathrm{w}$ and $17.16 \%$ w/w respectively. This gave a $\mathrm{Ca} / \mathrm{P}$ weight ratio of 2.15 or $1.67 \pm 0.02$ in molar ratio. The $\mathrm{Ca}$ to $\mathrm{P}$ weight ratio of the HAC powder gave a value of 2.14 or a molar ratio of $1.66 \pm$ 0.02 . These values are in agreement with that of the theoretical stoichiometry value of 1.667. This result suggests that the powders used are free from residual phases of tricalcium phosphates, calcium carbonate and calcium oxide. In general, the presence of any of these residual phases would give a $\mathrm{Ca} / \mathrm{P}$ ratio which has a value that deviates from the stoichiometry value of 1.667 . Achieving a stoichiometric $\mathrm{Ca} / \mathrm{P}$ molar ratio is of paramount importance, as any deviation from the stoichiometry value affects the solubility, osteocompatability, densification and mechanical properties of the 
hydroxyapatite ceramics $[13,14]$. The morphology of the precursor powders is shown in Figure 1. Both powders show a broad range of particle size distribution. The distribution of the HAS powder is bimodal with two peaks at $4 \mu \mathrm{m}$ and $33 \mu \mathrm{m}$. The mean and median of the distribution are $18.4 \mu \mathrm{m}$ and $15.1 \mu \mathrm{m}$ with $90 \%$ of the particles being below 40.3 $\mu \mathrm{m}$. The average powder particle size of the HAC powder was measured to be about 11 $\mu \mathrm{m}$ with a median of about $6 \mu \mathrm{m}$. Although the HAC powder is composed of finer particles, the degree of agglomeration is higher compared to the HAS powder.

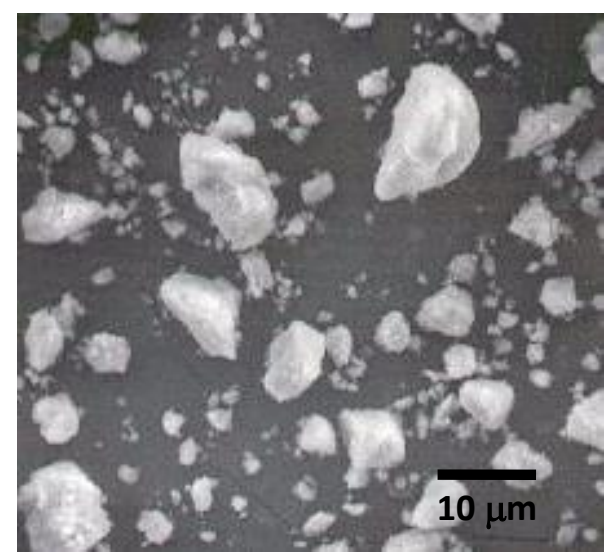

(a) HAS

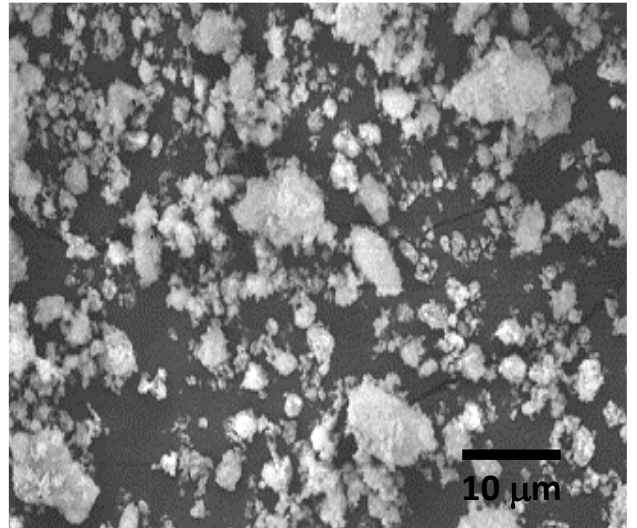

(b) HAC

Figure 1. Scanning electron micrographs of HA powders prepared from different sources.

\section{Phase Retention}

The crystalline phases identified for the samples sintered at various temperatures are given in Table 1.

Table 1. Phases detected in samples sintered at various temperatures.

\begin{tabular}{ccc}
\hline $\begin{array}{c}\text { Sintering temperature } \\
\left({ }^{\circ} \mathrm{C}\right)\end{array}$ & \multicolumn{2}{c}{ Distinguishable phases by XRD } \\
\cline { 2 - 3 } 1000 & HAC & HAS \\
1050 & HA & HA \\
1100 & HA & HA \\
1150 & HA & HA \\
1200 & HA & HA \\
1250 & HA & HA \\
1300 & HA & HA, TTCP, CaO \\
1350 & HA & HACP, CaO \\
1400 & HA & HA, $\alpha$-TCP, $\beta-\mathrm{TCP}, \mathrm{TTCP}$, \\
1450 & HA, $\alpha-\mathrm{TCP}$ & - \\
& HA, $\alpha-\mathrm{TCP}, \beta-\mathrm{TCP}, \mathrm{TTCP}$, & $\mathrm{CaO}$ \\
\hline
\end{tabular}


The HAS samples were found to be stable until a temperature of $1250^{\circ} \mathrm{C}$ with no residual phases detected. Decomposition of HA began to be observed in samples sintered at $1300^{\circ} \mathrm{C}$, with the formation of tetra-calcium phosphate (TTCP) and calcium oxide $(\mathrm{CaO})$, as depicted in Figure 2. However, as the sintering temperature was increased above $1300^{\circ} \mathrm{C}$, the intensity of peaks corresponding to these secondary phases also increased, indicating that further decomposition had occurred. Sintering at $1400^{\circ} \mathrm{C}$ resulted in the formation of $\alpha$-TCP and $\beta$-TCP in the HAS sample. Compacts sintered beyond this temperature were found to melt, forming glassy phases.

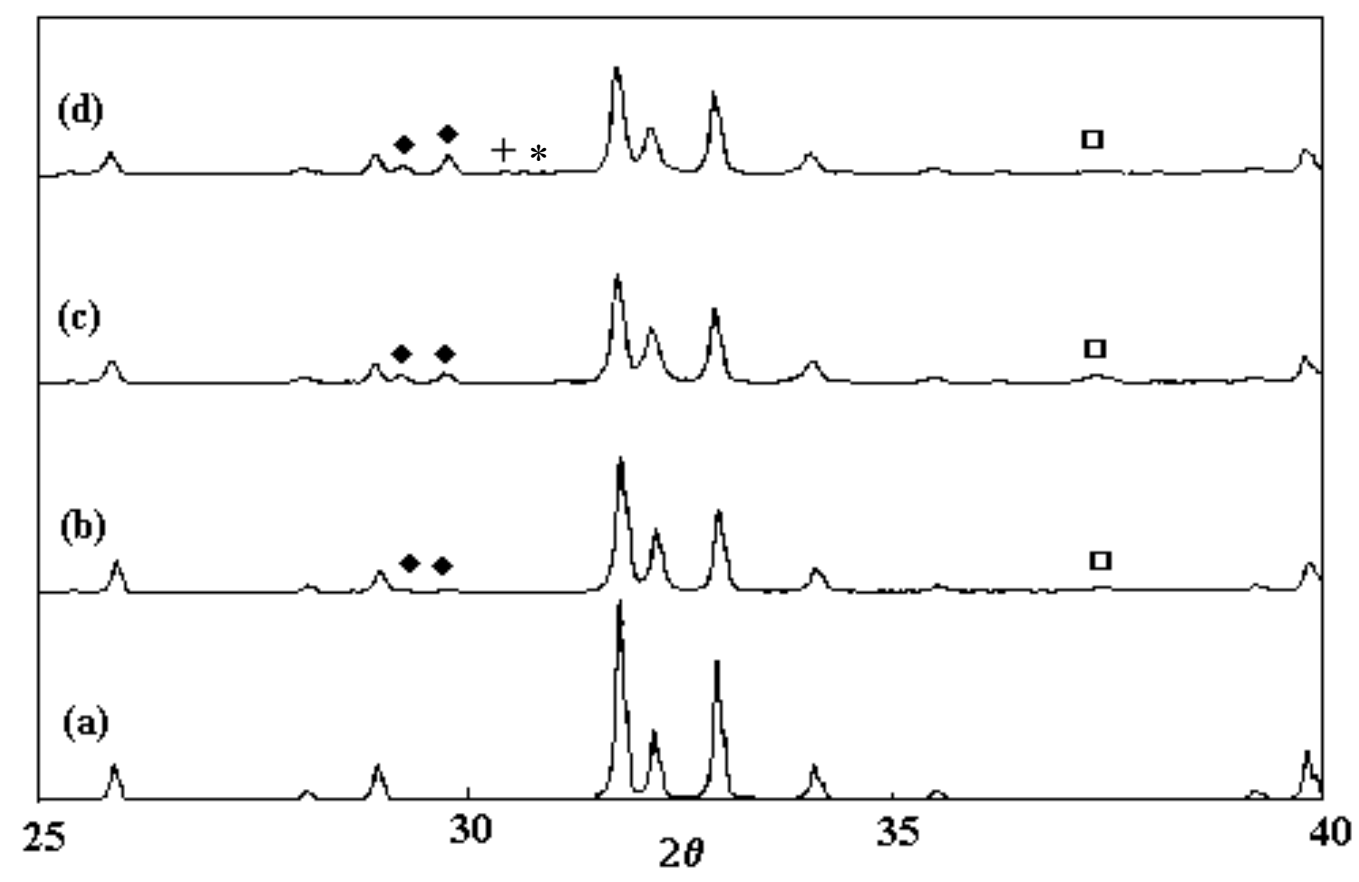

Figure 2. X-ray diffraction patterns of HAS samples sintered for 2 hours at (a) $1250^{\circ} \mathrm{C}$, (b) $1300^{\circ} \mathrm{C}$, (c) $1350^{\circ} \mathrm{C}$ and (d) $1400^{\circ} \mathrm{C}$. All the peaks in (a) correspond to the HA phase. (Key: $\diamond=\mathrm{TTCP} ;+=\alpha-\mathrm{TCP} ; *=\beta-\mathrm{TCP}$ and $\boldsymbol{\square}=\mathrm{CaO}$ ).

When sintered at temperatures below $1400^{\circ} \mathrm{C}$, the HAC samples consisted solely of HA phase, as shown in Figure 3. The formation of tri-calcium phosphate (TCP) was detected in the $\mathrm{HAC}$ samples sintered at $1400^{\circ} \mathrm{C}$. A further rise in the sintering temperature to $1450^{\circ} \mathrm{C}$ led to further $\mathrm{HA}$ decomposition, with the formation of tetracalcium phosphate (TTCP) and calcium oxide $(\mathrm{CaO})$ phases (see Figure 3 ). These findings indicate that the decomposition of HA is governed by the following equations $[15,16]$ :

$$
\begin{aligned}
& \mathrm{Ca}_{10}\left(\mathrm{PO}_{4}\right)_{6}(\mathrm{OH})_{2} \rightarrow 2 \mathrm{Ca}_{3}\left(\mathrm{PO}_{4}\right)_{2}+\mathrm{Ca}_{4} \mathrm{P}_{2} \mathrm{O}_{9}+\mathrm{H}_{2} \mathrm{O} \\
& \mathrm{Ca}_{10}\left(\mathrm{PO}_{4}\right)_{6}(\mathrm{OH})_{2} \rightarrow 3 \mathrm{Ca}_{3}\left(\mathrm{PO}_{4}\right)_{2}+\mathrm{CaO}+\mathrm{H}_{2} \mathrm{O}
\end{aligned}
$$

It is evident that the decomposition of the HAS samples started to occur at a lower temperature $\left(1300^{\circ} \mathrm{C}\right)$ than in the HAC samples $\left(1400^{\circ} \mathrm{C}\right)$. The different morphology of the latter powder was believed to have delayed the decomposition rate by preventing dehydration of the $\mathrm{OH}$ group from the HA matrix. In the present work, the decomposition of HAC samples proceeded according to Eq. (1) followed by Equation (2) for samples 
sintered at higher temperatures. On the contrary, the HAS samples seemed to decompose according to Eq. (2) first, with the formation of $\mathrm{CaO}$. At this stage, this phenomenon is unclear and further work is required to elucidate the decomposition behaviour of HAS material.

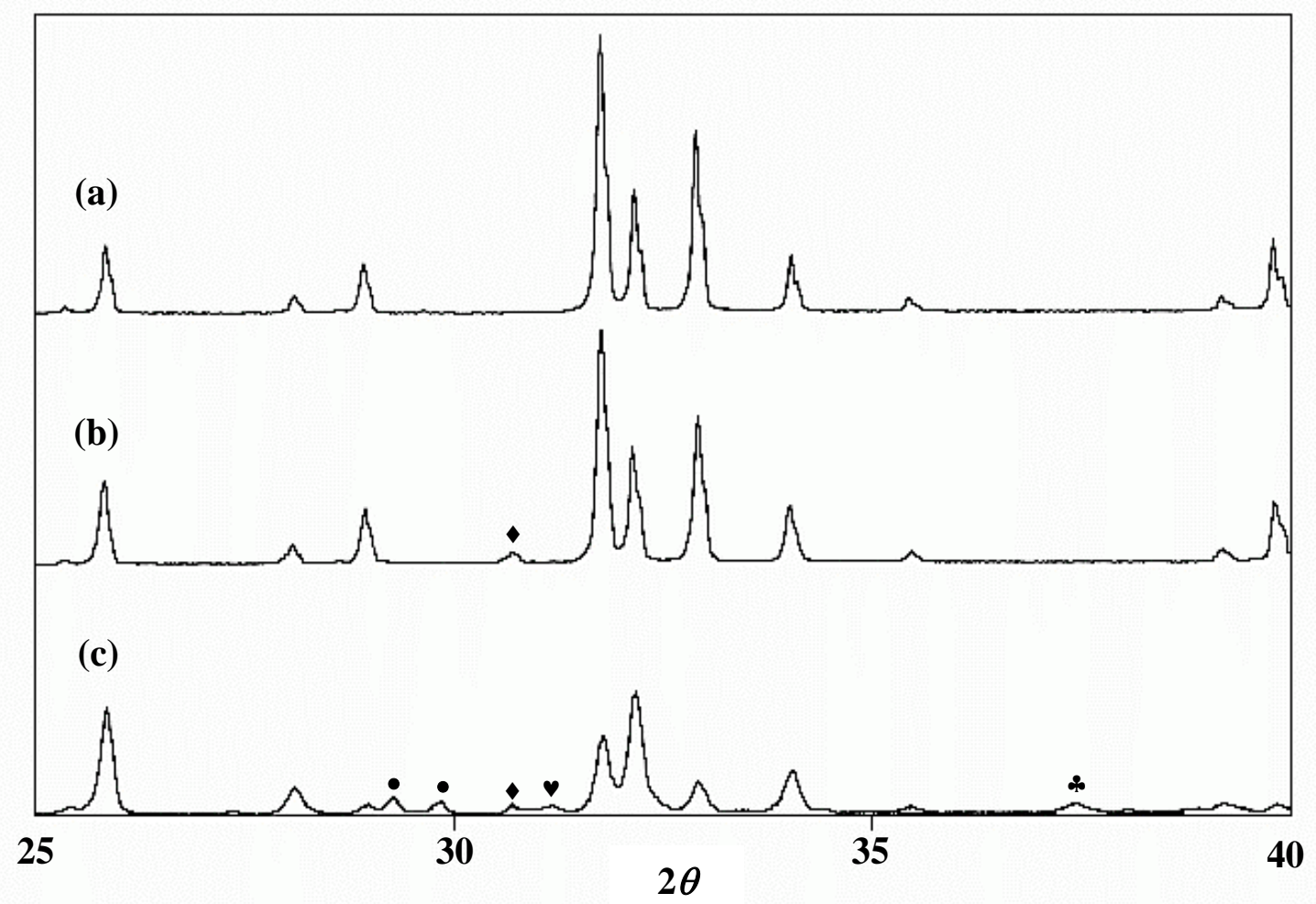

Figure 3. X-ray diffraction patterns of HAC sintered for 2 hours at (a) $1250^{\circ} \mathrm{C}$,

(b) $1400^{\circ} \mathrm{C}$ and (c) $1450^{\circ} \mathrm{C}$. All the peaks in (a) correspond to the HA phase.

$($ Key: $\bullet=$ TTCP; $\bullet=\alpha$-TCP; $\bullet=\beta-$ TCP and $\bullet=\mathrm{CaO}$ ).

\section{Density}

The effect of sintering temperatures on the relative density of HA is shown in Figure 4. For low temperature firing $\left(<1200^{\circ} \mathrm{C}\right)$, the HAS samples exhibited a slight variation in the relative density, i.e., from $98.1 \%$ at $1000^{\circ} \mathrm{C}$ to $>99 \%$, with the highest value of $99.8 \%$ being attained for samples sintered at $1150^{\circ} \mathrm{C}$. However, the relative density of the HAS samples was observed to decline slightly from $99.8 \%$ to $98.7 \%$ as the sintering temperature was increased from $1150^{\circ} \mathrm{C}$ to $1400^{\circ} \mathrm{C}$ (see Figure 4). In general, the variation of density with sintering temperature for the HAS material is small compared to the HAC samples. As can be observed in Figure 4, the HAC samples showed a significant difference in density over the sintering temperature employed. It was found that samples sintered at $1000^{\circ} \mathrm{C}$ exhibited the lowest relative density of $77 \%$, while the highest value of $99.8 \%$ was attained in HA sintered at $1400^{\circ} \mathrm{C}$. The low bulk density of the samples sintered at lower temperatures can be attributed to the low densification temperature employed, which resulted in the presence of residual porosity located mainly at grain boundaries $[12,17]$. 


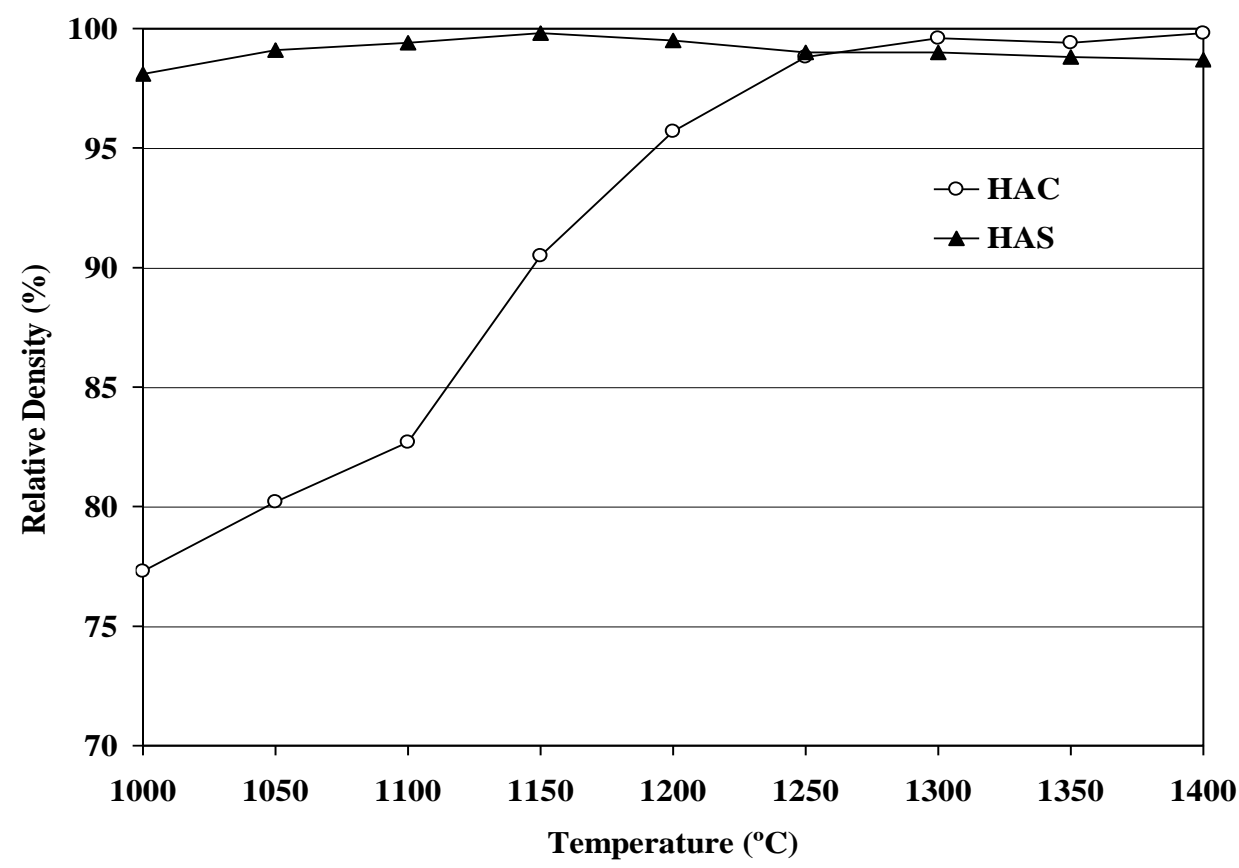

Figure 4. Variation in relative density of HAS and HAC samples with sintering temperature.

\section{Hardness}

The change in Vicker's hardness of the sintered HA samples with increasing sintering temperature is presented in Figure 5.

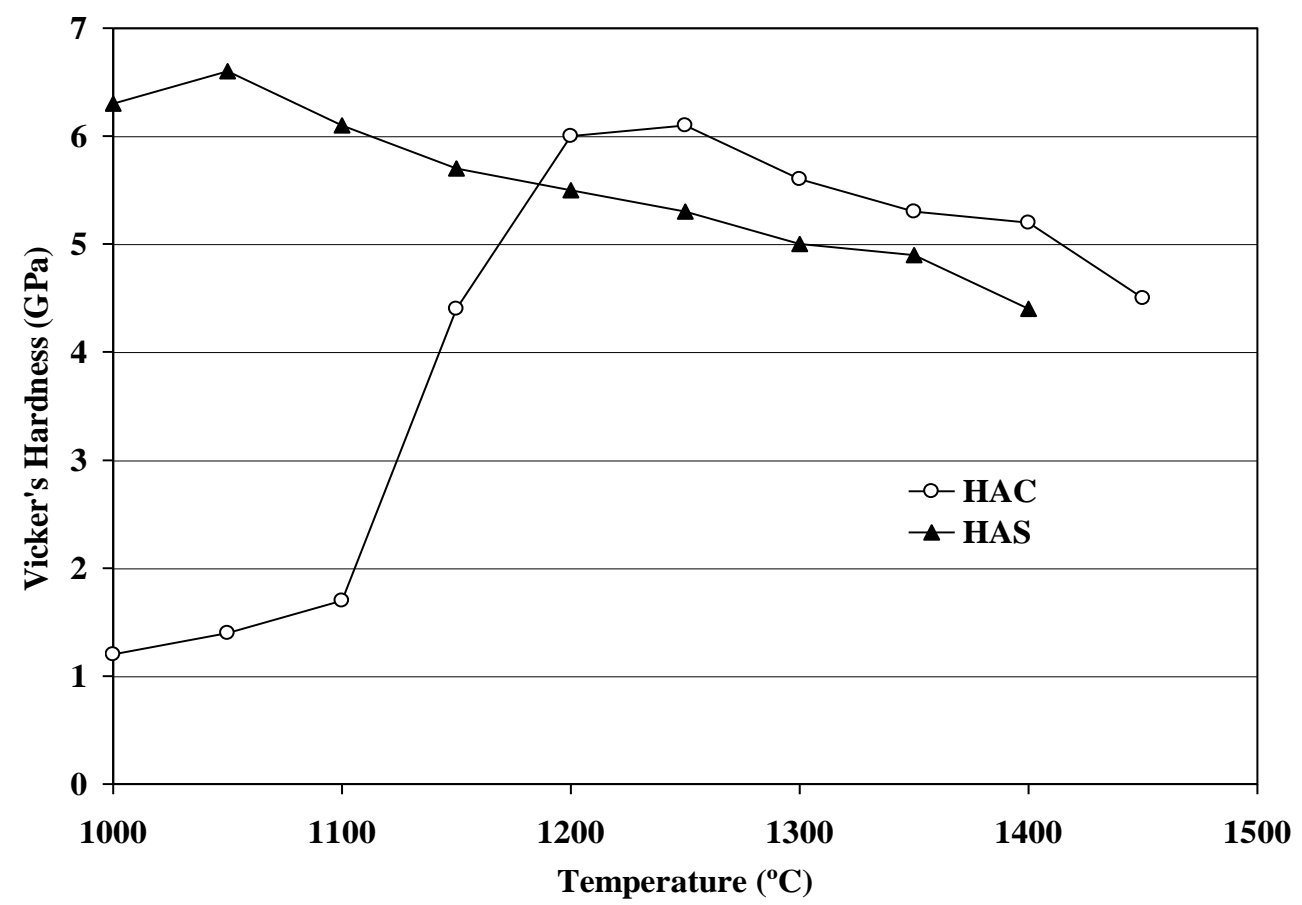

Figure 5. Variation in relative density of HAS and HAC with sintering temperature. 
It was found that the $\mathrm{HAC}$ samples sintered at $1250^{\circ} \mathrm{C}$ exhibited the highest hardness, i.e. $6.08 \pm 0.28 \mathrm{GPa}$, while the HAS sample sintered at $1050^{\circ} \mathrm{C}$ gave the highest value of $6.64 \pm 0.28 \mathrm{GPa}$. However, for both materials, as the sintering temperature increased the hardness was observed to decrease, despite having relatively high density values of more than 99\%. Previously, it has been proposed that the decline in hardness with increasing sintering temperature is associated with a grain size effect, i.e., above a certain critical grain size, the hardness of HA will decrease [12].

\section{CONCLUSIONS}

In the present work, the sintering behaviour of two stoichiometric HA powders was investigated. The study revealed that the decomposition of the HA powders was dependent on the morphology of the precursor powders. The synthesised powder (HAS) was found to achieve full densification at a lower temperature $\left(1150^{\circ} \mathrm{C}\right)$ than the commercially available HA powder $(\mathrm{HAC})\left(1400^{\circ} \mathrm{C}\right)$. However, the former sample also decomposed at a lower temperature to form various residual phases. It is believed that the difference in the level of agglomeration observed in the powders was responsible for the difference in the sintering characteristics.

\section{ACKNOWLEDGEMENTS}

This study was supported under the UM PPP grant No. PG079-2013A.

\section{REFERENCES}

[1] Sopyan I, Ramesh S, Nawawi N, Tampieri A, Sprio S. Effects of manganese doping on properties of sol-gel derived biphasic calcium phosphate ceramics. Ceramics International. 2011;37:3703-15.

[2] Kamiya K, Yoko T, Tanaka K, Fujiyama Y. Growth of fibrous hydroxyapatite in the gel system. Journal of Materials Science. 1989;24:827-32.

[3] Tan CY, Singh R, Teh YC, Tan YM, Yap BK. The effects of calcium-tophosphorus ratio on the densification and mechanical properties of hydroxyapatite ceramic. International Journal of Applied Ceramic Technology. 2015;12:223-7.

[4] $\mathrm{Wu} \mathrm{JM}$, Yeh TS. Sintering of hydroxylapatite-zirconia composite materials. Journal of Materials Science. 1988;23:3771-7.

[5] Sharif NS, Mohamed JJ, Derita HS, Ahmad ZA, Abdullah MZ, Mohamad H, et al. The effect of sintering conditions on the microstructure and electrical properties of $\mathrm{Pb}(\mathrm{Zr} 0.52 \mathrm{Ti0.48}) \mathrm{O} 3$ ceramic. Journal of Mechanical Engineering and Sciences. 2014;6:901-6.

[6] Ozeki K, Fukui Y, Aoki H. Influence of the calcium phosphate content of the target on the phase composition and deposition rate of sputtered films. Applied surface science. 2007;253:5040-4.

[7] Ramesh S, Tan C, Sopyan I, Hamdi M, Teng W. Consolidation of nanocrystalline hydroxyapatite powder. Science and Technology of Advanced Materials. 2007;8:124-30.

[8] Ellis SK, McNamara Jr EP. Powder synthesis research at CAMP. American Ceramic Society bulletin. 1989;68:988-94. 
[9] Sivakumar S, Ramesh S, Chin KL, Tan CY, Teng WD. Effect of sintering profiles on the properties and ageing resistance of Y-TZP ceramic. International Journal of Automotive and Mechanical Engineering. 2011;4:405-13.

[10] Ramesh S, Tan CY, Amiriyan M, Teng WD. Sintering effects on the densification of nanocrystalline hydroxyapatite. International Journal of Automotive and Mechanical Engineering. 2011;3:249-55.

[11] Ramesh S, Christopher P, Tan C, Teng W. The effect of cold isostatic pressing on the sinterability of synthesized HA. Biomedical Engineering: Applications, Basis and Communications. 2004;16:199-204.

[12] Murray M, Wang J, Ponton C, Marquis P. An improvement in processing of hydroxyapatite ceramics. Journal of Materials Science. 1995;30:3061-74.

[13] Best S, Bonfield W. Processing behaviour of hydroxyapatite powders with contrasting morphology. Journal of Materials Science: Materials in Medicine. 1994;5:516-21.

[14] Wang PE, Chaki T. Sintering behaviour and mechanical properties of hydroxyapatite and dicalcium phosphate. Journal of Materials Science: Materials in Medicine. 1993;4:150-8.

[15] Liu H, Chin T, Lai L, Chiu S, Chung K, Chang C, et al. Hydroxyapatite synthesized by a simplified hydrothermal method. Ceramics International. 1997;23:19-25.

[16] Ogiso M, Nakabayashi N, Matsumoto T, Yamamura M, Lee RR. Adhesive improvement of the mechanical properties of a dense HA-cemented Ti dental implant. Journal of biomedical materials research. 1996;30:109-16.

[17] Muralithran G, Ramesh S. The effects of sintering temperature on the properties of hydroxyapatite. Ceramics International. 2000;26:221-30. 